\title{
Measurement of interplanetary magnetic field in short period using the cosmic-ray Sun shadow measured by LHAASO.
}

Yuncheng Nan ${ }^{a, b, *}$, Songzhan Chen $^{b}$ and Cunfeng Feng ${ }^{a}$ on behalf of the LHAASO Collaboration

(a complete list of authors can be found at the end of the proceedings)

${ }^{a}$ Shandong University, Institute of Frontier and Interdisciplinary Science,72, Binhai Road, Jimo, Qingdao, Shandong, China

${ }^{b}$ Key Laboratory of Particle Astrophysics, Institute of High Energy Physics, CAS, 19-yi, Yuquan Road, Shijingshan District, Beijing, China

E-mail: nanyc@ihep.ac.cn

The interplanetary magnetic field (IMF) between the Sun and the Earth induces the displacement of the cosmic-ray Sun shadow from the optical position. Previously, the average IMF has been measured by the ARGO-YBJ and the Tibet-AS $\gamma$ experiments through several years of data. With the improvement of the sensitivity, the first pool of WCDA in LHAASO, which has been operated since April 2019, and has observed the Sun shadow with significance exceeds 70 standard deviation. Using the data collected by WCDA from July 26 to August 22, 2019, we measured the displacements of Sun shadow at the energy of $6.4 \mathrm{TeV}$ every two or three days. Combining with the simulation of Sun shadow, the IMF is measured and is comparable with the satellite observations. This is the first time to measure the IMF using Sun shadow in a short period, and the expectation for space weather forecast is discussed.

$37^{\text {th }}$ International Cosmic Ray Conference (ICRC 2021)

July 12th - 23rd, 2021

Online - Berlin, Germany 


\section{Introduction}

The photosphere magnetic field of the Sun is blown out by the solar wind and reaches the interplanetary space, forming the interplanetary magnetic field(IMF)[1]. It is very wide from several solar radii to the Earth (about 250 solar radii), which makes the regular measurement of IMF very complicated. When the solar magnetic field is stable, the coronal hole will emit highspeed solar wind, and the coronal hole high-speed flow will be formed in interplanetary[2]; In addition to the stable magnetic activity cycle, in the Sun's explosive period, the coronal ejections of matter into the interplanetary space[3]. At present, there is no direct observation method for these magnetic fields and their variation, usually using satellites, such as SOHO $\backslash$ LASCO. Some of these two activities reach the Earth, form geomagnetic storms and affect human life. Therefore, it is very important to measure and forecast them more.

Cosmic rays originating outside the solar system usually arrive at the Earth nearly isotropically. In the process, they may be blocked by the Sun or the Moon and casts a shadow on the sky map. The main component of the cosmic rays is the positive charged nuclei. Before they travels in the universe before arriving at the Earth, they are deflected by the Lorentz force and became probes of the magnetic field. Therefore, the shadows can be used to measure the whole solar-terrestrial space magnetic field and its variation[4].

In the past 30 years, Sun shadow has been observed by many experiments, such CYGNUS, Tibet-AS $\gamma$, Milagro, ARGO-YBJ, HAWC, IceCube, etc. In addition to the study of the solar magnetic field and its activities researched by Tibet-AS $\gamma$ [5][6][7], the study of the interplanetary magnetic field has also become a challenge for many Sun shadow observation experiments. In 2000, Tibet-AS $\gamma$ observed and simulated the Sun shadow in solar cycle 22, and found the relationship between the displacements of the Sun shadow along the south or north direction and the two fanshaped structures "away" or "toward" of the IMF[8]. In 2011, ARGO-YBJ experiment measured the y-component of the IMF in solar cycle 23-24 through the displacement of Sun shadow along the south or north direction and found that the IMF observed by the Sun shadow was 1.6 days ahead of that observed by the satellite, which has a potential forecasting capability for magnetic storms due to explosive solar activity[9]. But it is limited by their observation sensitivity. In 2018, Tibet-AS $\gamma$ also quantitatively measured the average IMF in solar cycle 23 through further comparison between the Sun shadow data and the simulation[10]. In 2019, Tibet-AS $\gamma$ also proposed for the first time to estimate the average z-component of the IMF by using the east-west shift of the Sun shadow, which is related to the generation of geomagnetic storms[11].

According to the research above, due to the limitation of sensitivity, the IMF that originally changed with the solar magnetic field all the time can only be measured by its mean value or changes by the annual level data. The Large High Altitude Air Shower Observatory (LHAASO) is a newgeneration complex EAS array being constructed at Daocheng, Sichuan province, China $\left(100.01^{\circ} \mathrm{E}\right.$, $29.35^{\circ} \mathrm{N}$ ). The first pool of Water Cherenkov Detector Array (WCDA-1) is one of the main detector arrays in LHAASO to survey transient phenomena and discovering new sources[12] and has been operated since April 2019. Recently, Crab, as standard candlelight has been observed[13], and Moon shadow has monthly significance up to $13-22 \sigma[14]$. In this work, we will measure the IMF by combining the data with the simulation of Sun shadow of WCDA-1. The expectation of space weather forecast for solar activities will also be discussed. 


\section{Experiment and data analysis}

WCDA- 1 is a square pond of $150 \mathrm{~m} \times 150 \mathrm{~m}$ consisting of 900 detector units. At the bottom of each detection unit, there is an 8-inch photomultiplier tube to measure the Cherenkov light produced by cosmic ray secondary particles in water. By recording the arrival time, total charge and so on, we can get the information of the primary cosmic ray particles. WCDA-1 has been successfully operated since April 2019. For these collected data, we use the off-line characteristic plane, the centre-of-gravity, and fitting the shower plane formed by secondary particles with a cone methods to calibrate the arrival time, core position of secondary particles and the direction of the primary event. At the same time, the number of fire detection units after filtering out the noise is recorded, as $N$ fit. More details about the detector, calibration and reconstruction can be found in [15][16].

To use the best data to observe the IMF, we selected the data: (1)The noise data caused by other calibration and the data of detection unit not working are excluded; (2)Good agreement between experimental data and simulation data: $N$ fit : 100 - 800; (3)The distance between the sun and the moon is required to more than $5^{\circ}$ to remove the overlapping effects; (4)The complete time range in which highly significant solar shadow can be observed: July 26 to August 22, 2019 (CR2220); (5)Zenith angle less than $50^{\circ}$.

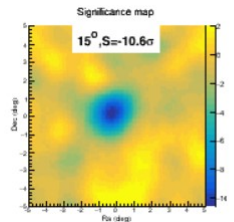

nimats
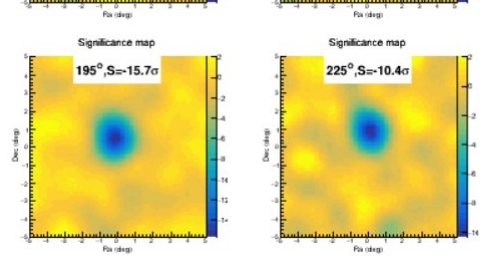
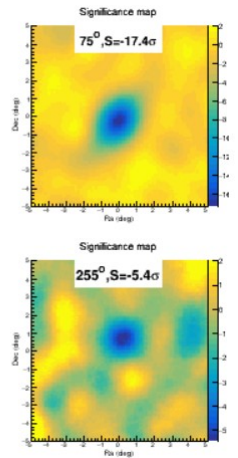
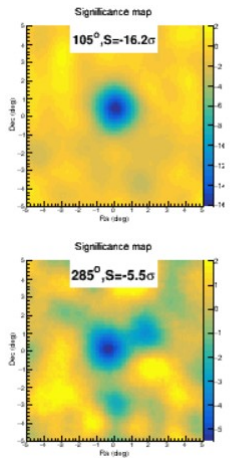
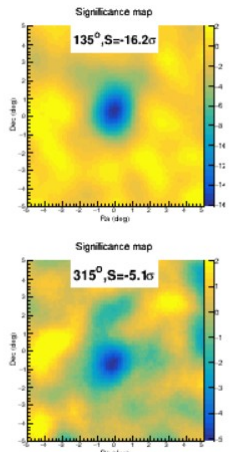
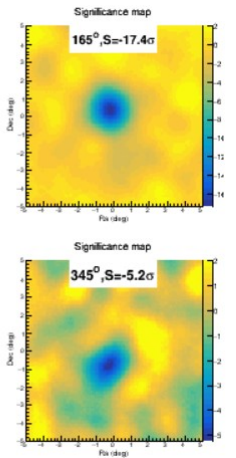

Figure 1: Significant map of Sun shadow at different carrington longitudes. $E=6.2 \mathrm{TeV}$

For the Sun shadow of CR2220, the data set is divided into 12 groups on average according to the carrington longitude. For each data set, the $20^{\circ} \times 20^{\circ}$ sky map in celestial coordinates (right ascension RA and declination Dec.) is built with $0.1^{\circ} \times 0.1^{\circ}$ bin size. Fixing the $(0,0)$ of the map at the center of the Sun, the number of the observed events in each bin is recorded to the $N_{\text {on }}$. The corresponding background in each bin is estimated by the direct integral method which is used[16] where we called it $N_{b}$. The background events are subtracted from the total observed events to obtain the signal events $N_{s}$. Then we calculated the statistical significance of these signals by the Li\&Ma's formula(9)[17]. With taking into account the array angular resolution(Rsmooth), the significance maps for different data set are shown in Figure 1.

The displacement of the Sun shadow in the north-south direction is obtained by 1D projection of the sky map of the signal events $N_{s}$. Gaussian fitting is used to get the peak position of the distribution, namely mean value and it's error. The displacements of the Sun shadow in the northsouth direction and it's error at different carrington longitudes is summarized in Figure 2. With the same method, we get that the pointing accuracy of the detector by the observing Moon Shadow along 
the north and south direction is $-0.01^{\circ} \pm 0.01^{\circ}$, which the degree of accuracy of the deflections of the Sun shadow shown in Figure 2, and that the angular resolution is $0.56^{\circ}$.

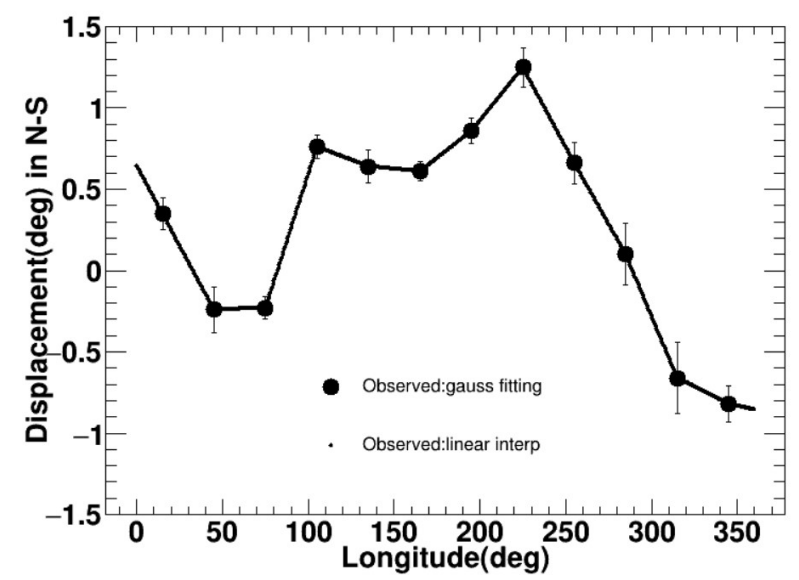

Figure 2: The displacements of the Sun shadow in the north-south direction at different carrington longitudes. The small black dots represent the result obtained from the linear interpolation of the fitting results of 12 data samples.

\section{MC simulation}

The cosmic ray simulation data, including $\mathrm{H}, \mathrm{He}, \mathrm{C}-\mathrm{N}-\mathrm{O}, \mathrm{Mg}-\mathrm{Al}-\mathrm{Si}$, and $\mathrm{Fe}$, is generated by the air shower simulation software CORSIKA(v75000) and the detector response software G4WCDA[18]. The energy range is from $100 \mathrm{GeV}$ to $1 \mathrm{PeV}$ and the zenith angle range is from $0^{\circ}$ to $60^{\circ}$ for the simulation for the Sun shadow. Also, using the selection conditions of experimental data, we get the information of cosmic rays that can be observed by experiments, including the composition, primary energy, and the number of events, as shown in Figure 3.

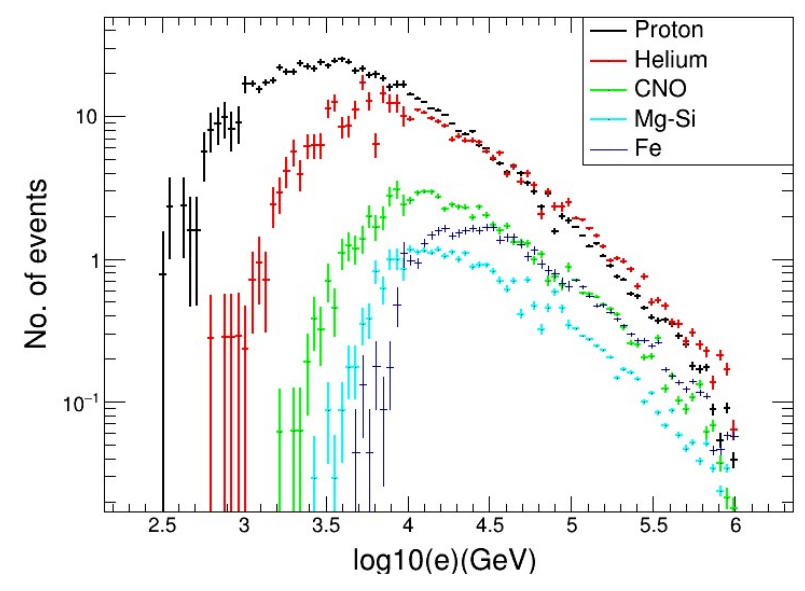

Figure 3: Energy distribution of cosmic rays with different components.

The simulation of Sun shadow outside the atmosphere includes the simulation of the signal and the background. The background simulation of the Sun shadow is divided into two steps. First, we get the number of background $N_{b}$ and its distribution with declination at the Sun's position 
from the experimental data of Sun shadow. Second, according to the principle of direct integration method and using the cosmic ray information in Figure 3, we simulated the background by the back forward method[19]. When the $\mathrm{B}=0$, we record the position of the particles in the sky map and get the background map of the Sun shadow. By using the same method, considering the influence of magnetic fields, if the particles hit the Sun, we record the position of the missing "Sun shadow events" and obtained the $N_{s}$ map. Finally, we sample the Gaussian distribution of $N_{s}$ in each bin to simulate the effect of angular resolution which is due to the detector response.

In this simulation process, the solar-terrestrial space magnetic field, including coronal magnetic field, IMF, and geomagnetic field, needs to be calculated accurately. For the coronal magnetic field, we use the photosphere magnetograms from GONG[20], and the magnetic field extrapolation model is the Potential Field Source Surface (PFSS)[21], where the source surface $\left(R_{S S}\right)=2.5 R_{\odot}$. And the order of spherical harmonics expansion $\mathrm{n}$ is set to 9. For the IMF, we use the modified Parker's model where is used in ARGO-YBJ's paper[9]:

$$
\begin{aligned}
& B_{r}(r, \theta, \phi)=B_{0} f\left(\phi_{0}-\delta\right)\left(\frac{R_{S S}}{r}\right)^{2} \\
& B_{\theta}(r, \theta, \phi)=0 \\
& B_{\phi}(r, \theta, \phi)=B_{0} f\left(\phi_{0}-\delta\right)\left(\frac{\omega}{v}\right)\left(r-R_{S S}\right)\left(\frac{R_{S S}}{r}\right)^{2} \sin \theta \\
& \frac{r}{R_{S S}}-1-\ln \left(\frac{r}{R_{S S}}\right)=\frac{v}{\omega R_{S S}}\left(\phi-\phi_{0}\right)
\end{aligned}
$$

where the $\omega$ is the angular velocity of the sun, approximately equal to $2.7 \pm 10^{-6} \mathrm{rad} / \mathrm{s}$. And the $\mathrm{v}$ and $\mathrm{r}$ are the radial component of the solar wind velocity where we used comes from OMNI observation and the distance to the Sun, respectively. $B_{0}$ and function $\mathrm{f}\left(\phi_{o}-\delta\right)$ are the magnitude of the magnetic field at $R_{s s}$ and it's variation with carrington longitude which we have observed by Sun shadow in Figure 2. $\delta$ is a shift of the carrington longitute $\phi_{o}$, taking into account the cumulative and average effect of the IMF magnetic field measured by cosmic rays. $B_{0}$ and $\delta$ are the two parameters that can be estimated with our data. We use the international geomagnetic reference field-12[22] to describing the GMF. When $B_{0}=0.1$ Gauss and $\delta=0$, we have carried out the preliminary simulation and compared it with the observation, as shown in Figure 4.
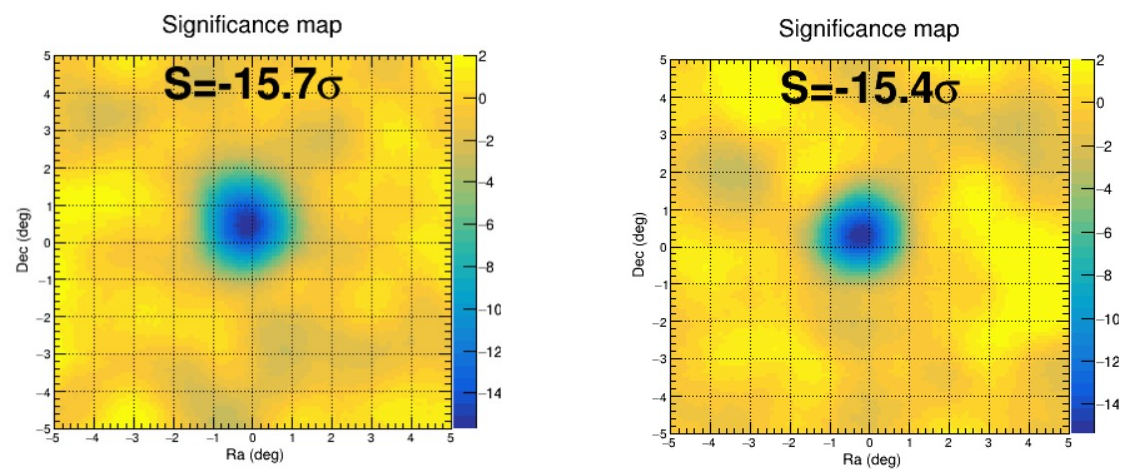

Figure 4: The observed and the simulated significance map of the Sun shadow when the carrington longitude equals $195^{\circ}$. 


\section{Method of IMF measurement}

We have observed the displacement of the Sun shadow along the north-south direction with the change of carrington longitude, as shown in Figure 2, which represents the magnitude of the IMF at the source surface and the spiral part of the magnetic field line caused by the solar wind and Sun's rotation at a certain carrington longitude. The observation of the Sun shadow is a cumulative and average effect. In order to determine this unknown carrington longitude, ARGO-YBJ[9] introduced parameter $\delta$ and used parameter $B_{0}$ to correct the magnitude of the IMF at the source surface.

The parameters $\delta$ and $B_{0}$ are obtained by comparing the observed and simulated deflections of Sun shadow along the north-south direction. In order to improve the simulation speed, five groups of simulation data with $\delta=0, B_{0}$ from 0.05 to 0.25 Gauss, with an interval of 0.05 , are calculated. The simulation results and the experimental results in Figure 2 are summarized in Figure 5. The best-fit values of $\delta$ and $B_{0}$ are planned to be obtained by minimizing a $\chi^{2}$ function for 12 carrington longitudes bins:

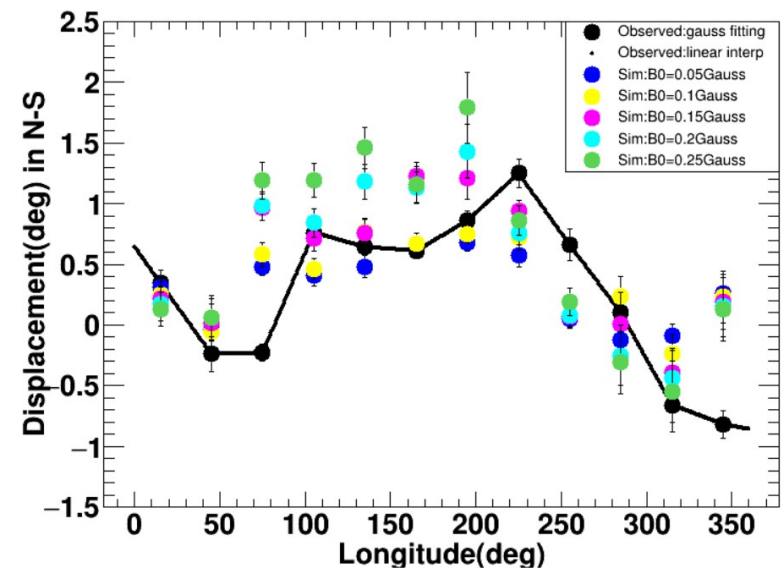

Figure 5: The observed and simulated displacements of the Sun shadow in the north-south direction at different carrington longitudes. The color dots represent the simulated results for different $\boldsymbol{B}_{0}$.

$$
\chi^{2}=\sum_{i=1}^{12} \frac{\left(D_{s i m}^{i}\left(\delta, B_{0}\right)-D_{o b s}^{i}\right)^{2}}{\sigma_{s i m}^{2}+\sigma_{o b s}^{2}},
$$

where D represents the displacement value in Figure 5. And D for the certain $\delta$ and $B_{0}$ can be obtained by $2 \mathrm{D}$ linear interpolation.

\section{Result and discussion}

According to the fitting method in the previous part, we get the value of $\delta=44.98^{\circ} \pm 0.00^{\circ}, B_{0}=$ $(0.20 \pm 0.00)$ Gauss. The value of $\chi^{2}=4.25$. And we measured the y-component of the IMF, as shown in Figure 6.

From Figure 6, we can see that the results of the Sun shadow measurement are in good agreement with those of the satellite measurement. In this work, the time of the IMF observed by the Sun shadow is 3.4 days $\left(44.98^{\circ}\right)$ ahead of the time of the IMF reaching the earth, or the time 


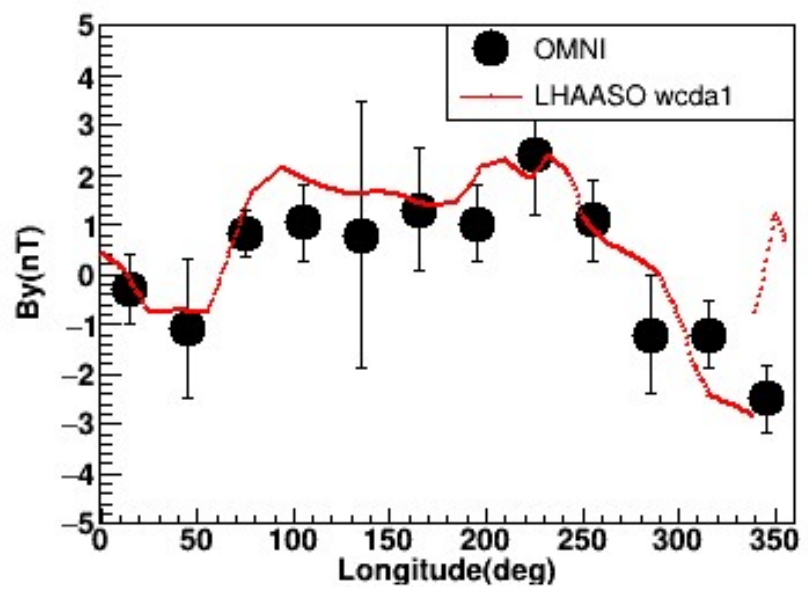

Figure 6: The y-components of the IMF measured by the LHAASO-WCDA-1 and the OMNI, respectively.

measured by satellite. This value is different from ARGO-YBJ's result of 1.6 days ahead[9]. We are still exploring the reason. It may come from the difference of solar wind speed or the problem of fitting. In addition, considering the actual observation, when the carrington longitude is equal to $195^{\circ}, 225^{\circ}$, and $255^{\circ}$, there is a fast flow from the coronal hole with a speed close to $700 \mathrm{~km} / \mathrm{s}$ flowing to and passing through the Earth. Because we are not sure about the magnetic field of this fast flow, we still insist on using Parker's model in this work, and we observed the y-component of the IMF at these three longitude positions. This may also be the reason why the time of Sun shadow measurement calculated in this work is different from that measured in ARGO-YBJ.

\section{Conclusion}

Using the data from the LHAASO-WCDA-1, we measured the y-component of the IMF, and it is in good agreement with the results from the satellite. From our work, the IMF will be measured at Earth is 3.4 days ahead of that from the satellite. This result shows that we have a potential forecasting capability for space weather. But the value of ahead of time we get is different from that from ARGO-YBJ[9], and we are looking for the reason. On the other hand, in CR2220, we also measured the y-component of the IMF caused by the fast flow generated by the coronal hole. The Z-component of the IMF is what we are concerned about, which is related to geomagnetic storms. We have seen the y-component of the IMF in advance through the Sun shadow, in the future, we will expect to be able to observe the Z-component of the IMF and forecast geomagnetic storm activity.

\section{Acknowledge}

This work is supported by the National Key R\&D Program of China (No. 2018YFA0404201) and the Natural Sciences Foundation of China (No. 11575203 and No. 11775131).

\section{References}

[1] E. N. Parker, 1958, ApJ, 128, 664 
[2] S. R. Habbal, R. Woo et al, 1997, ApJ, 489, 1

[3] N. Gopalswamy, Y. Hanaoka et al, 1998, Geophys. Res. Lett., 25, 2485

[4] G. W. Clark, 1957, Phys. Rev. 108, 450

[5] M. Amenomori, X. J. Bi et al, 1996, ApJ, 464, 954

[6] M. Amenomori, X. J. Bi et al, 2013, PRL, 2013, 111, 011101

[7] M. Amenomori, X. J. Bi et al, 2018, ApJ, 860, 13

[8] M. Amenomori, X. J. Bi et al, 2000, ApJ, 541, 1051

[9] G. Aielli, C. Bacci, et al, 2011, ApJ, 729, 113

[10] M. Amenomori, X. J. Bi et al, 2018, PRL, 120, 031101

[11] M. Amenomori, X. J. Bi et al, 2019, 36nd International Cosmic Ray Conference

[12] Z. Cao, 2010, Chin. Phys. C, 34, 249

[13] F. A. Aharonian, Z. Cao, Chin. Phys. C, 2021

[14] Y. J. Wang, M. Zha et al, 2021, 37nd International Cosmic Ray Conference

[15] H. H. He, 2018, Radiation Detection Technology and Methods, 2, 7

[16] F. A. Aharonian, Z. Cao, 2021, Chin. Phys. C, 45, 025002

[17] T. P. Li, Y. Q. Ma, 1983, ApJ, 272, 317

[18] S. Agostinelli, et al, 2003, Nucl. Inst. Methods. Phys. Res. A, 506, 250

[19] Y. C. Nan, S. Z. Chen, 2019, 36nd International Cosmic Ray Conference

[20] https://gong.nso.edu/

[21] K. H. Schatten, J. M. Wilcox, N. F. Ness, 1969, Solar Physics, 6, 442

[22] https://www.ngdc.noaa.gov/IAGA/vmod/igrf.html 


\section{Full Authors List: LHAASO Collaboration}

Zhen $\mathrm{Cao}^{1,2,3}$, F. Aharonian ${ }^{4,5}$, Q. An ${ }^{6,7}$, Axikegu $^{8}$, L.X. Bai ${ }^{9}$, Y.X. Bai ${ }^{1,3}$, L.X. Bai ${ }^{9}$, Y.X. Bai ${ }^{1,3}$, Y.W. Bao ${ }^{10}$, D. Bastieri ${ }^{11}$, X.J. Bi ${ }^{1,2,3}$, Y.J. Bi ${ }^{1,3}$, H. Cai ${ }^{12}$, J.T. Cai ${ }^{11}$, Zhe Cao ${ }^{6,7}$, J. Chang ${ }^{13}$, J.F. Chang ${ }^{1,3,6}$, B.M. Chen ${ }^{14}$, E.S. Chen ${ }^{1,2,3}$, J. Chen ${ }^{9}$, Liang $\mathrm{Chen}^{1,2,3}$, Liang Chen ${ }^{15}$, Long $\mathrm{Chen}^{8}$, M.J. Chen ${ }^{1,3}$, M.L. Chen ${ }^{1,3,6}$, Q.H. $\mathrm{Chen}^{8}$, S.H. Chen ${ }^{1,2,3}$, S.Z. Chen ${ }^{1,3}$, T.L. Chen ${ }^{16}$,X.L. Chen $^{1,2,3}$, Y. Chen ${ }^{10}$, N. Cheng ${ }^{1,3}$, Y.D. Cheng ${ }^{1,3}$, S.W. Cui ${ }^{14}$, X.H. Cuii ${ }^{17}$, Y.D. Cui ${ }^{18}$, B. D'Ettorre Piazzoli1 ${ }^{19}$, B.Z. Dai ${ }^{20}$, H.L. Dai $^{1,3,6}$, Z.G. Dai ${ }^{7}$, Danzengluobu ${ }^{16}$, D. della Volpe ${ }^{21}$, X.J. Dong ${ }^{1,3}$, K.K. Duan ${ }^{13}$, J.H. Fan $^{11}$, Y.Z. Fan ${ }^{13}$, Z.X. Fan ${ }^{1,3}$, J. Fang ${ }^{20}$, K. Fang $^{1,3}$, C.F. Feng ${ }^{22}$, L. Feng ${ }^{13}$, S.H. Feng ${ }^{1,3}$, Y.L. Feng ${ }^{13}$, B. Gao ${ }^{1,3}$, C.D. Gao ${ }^{22}$, L.Q. Gao ${ }^{1,2,3}$, Q. Gao ${ }^{16}$, W. Gao ${ }^{22}$, M.M. Ge ${ }^{20}$, L.S. Geng ${ }^{1,3}$, G.H. Gong ${ }^{23}$, Q.B. Gou ${ }^{1,3}$, M.H. Gu ${ }^{1,3,6}$, F.L. Guo ${ }^{15}$, J.G. Guo ${ }^{1,2,3}$, X.L. Guo ${ }^{8}$, Y.Q. Guo ${ }^{1,3}$, Y.Y. Guo ${ }^{1,2,3,13}$, Y.A. $\mathrm{Han}^{24}$, H.H. He ${ }^{1,2,3}$, H.N. He ${ }^{13}$, J.C. He ${ }^{1,2,3}$, S.L. He ${ }^{11}$, X.B. He ${ }^{18}$, Y. He ${ }^{8}$, M. Heller ${ }^{21}$, Y.K. Hor ${ }^{18}$, C. Hou ${ }^{1,3}$, H.B. Hu ${ }^{1,2,3}$, S.

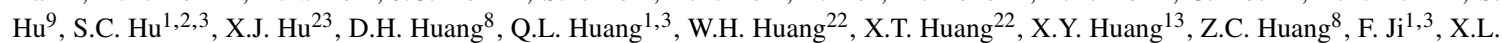
$\mathrm{Ji}^{1,3,6}$, H.Y. Jia ${ }^{8}$, K. Jiang ${ }^{6,7}$, Z.J. Jiang ${ }^{20}$, C. Jin ${ }^{1,2,3}$, T. Ke ${ }^{1,3}$, D. Kuleshov ${ }^{25}$, K. Levochkin ${ }^{25}$, B.B. Li ${ }^{14}$, Cheng Li ${ }^{6,7}$, Cong Li ${ }^{1,3}$,

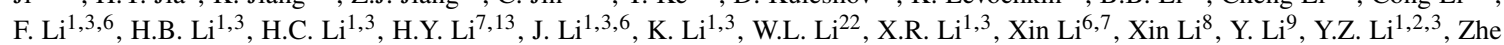

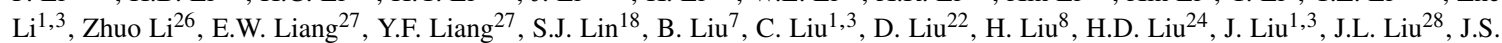

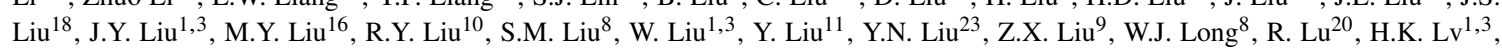

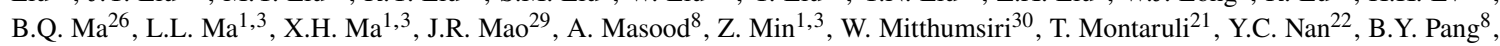
P. Pattarakijwanich ${ }^{30}$, Z.Y. Pei ${ }^{11}$, M.Y. Qi ${ }^{1,3}$, Y.Q. Qi ${ }^{14}$, B.Q. Qiao ${ }^{1,3}$, J.J. Qin ${ }^{7}$, D. Ruffolo ${ }^{30}$, V. Rulev ${ }^{25}$, A. Sáiz ${ }^{30}$, L. Shao ${ }^{14}$, O. Shchegolev $^{25,31}$, X.D. Sheng ${ }^{1,3}$, J.Y. Shi ${ }^{1,3}$, H.C. Song ${ }^{26}$, Yu.V. Stenkin ${ }^{25,31}$, V. Stepanov ${ }^{25}$, Y. Su ${ }^{32}$, Q.N. Sun ${ }^{8}$, X.N. Sun ${ }^{27}$, Z.B.

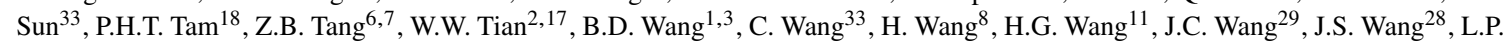
Wang $^{22}$, L.Y. Wang ${ }^{1,3}$, R.N. Wang ${ }^{8}$, W. Wang ${ }^{18}$, W. Wang ${ }^{12}$, X.G. Wang ${ }^{27}$, X.J. Wang ${ }^{1,3}$, X.Y. Wang ${ }^{10}$, Y. Wang ${ }^{8}$, Y.D. Wang ${ }^{1,3}$, Y.J. Wang $^{1,3}$, Y.P. Wang ${ }^{1,2,3}$, Z.H. Wang ${ }^{9}$, Z.X. Wang ${ }^{20}$, Zhen Wang ${ }^{28}$, Zheng Wang ${ }^{1,3,6}{ }^{1}$ D.M. Wei ${ }^{13}$, J.J. Wei ${ }^{13}$, Y.J. Wei ${ }^{1,2,3}$, T. Wen ${ }^{20}$, C.Y. Wu ${ }^{1,3}$, H.R. Wu ${ }^{1,3}$, S. Wu ${ }^{1,3}$, W.X. Wu ${ }^{8}$, X.F. Wu ${ }^{13}$, S.Q. Xi ${ }^{1,3}$, J. Xia ${ }^{7,13}$, J.J. Xia ${ }^{8}$, G.M. Xiang ${ }^{2,15}$, D.X. Xiao ${ }^{16}$, G. Xiao ${ }^{1,3}$, H.B. Xiao ${ }^{11}$, G.G. Xin ${ }^{12}$, Y.L. Xin ${ }^{8}$, Y. Xing ${ }^{15}$, D.L. Xu ${ }^{28}$, R.X. Xu ${ }^{26}$, L. Xue ${ }^{22}$, D.H. Yann ${ }^{29}$, J.Z. Yan ${ }^{13}$, C.W. Yang ${ }^{9}$, F.F. Yang ${ }^{1,3,6}$, J.Y. Yang ${ }^{18}$, L.L. Yang ${ }^{18}$, M.J. Yang ${ }^{1,3}$, R.Z. Yang ${ }^{7}$, S.B. Yang ${ }^{20}$, Y.H. Yao ${ }^{9}$, Z.G. Yao ${ }^{1,3}$, Y.M. Ye ${ }^{23}$, L.Q. Yin ${ }^{1,3}$, N. Yin ${ }^{22}$, X.H. You $^{1,3}$, Z.Y. You ${ }^{1,2,3}$, Y.H. Yu ${ }^{22}$, Q. Yuan ${ }^{13}$, H.D. Zeng ${ }^{13}$, T.X. Zeng ${ }^{1,3,6}$, W. Zeng ${ }^{20}$, Z.K. Zeng ${ }^{1,2,3}$, M. Zha ${ }^{1,3}$, X.X. Zhai ${ }^{1,3}$, B.B. Zhang $^{10}$, H.M. Zhang ${ }^{10}$, H.Y. Zhang ${ }^{22}$, J.L. Zhang ${ }^{1}$, J.W. Zhang ${ }^{9}$, L.X. Zhang ${ }^{11}$, Li Zhang ${ }^{20}$, Lu Zhang ${ }^{14}$, P.F. Zhang ${ }^{20}$, P.P. Zhang ${ }^{14}$, R. Zhang ${ }^{7,13}$, S.R. Zhang ${ }^{14}$, S.S. Zhang ${ }^{1,3}$, X. Zhang ${ }^{10}$, X.P. Zhang ${ }^{1,3}$, Y.F. Zhang ${ }^{8}$, Y.L. Zhang ${ }^{1,3}$, Yi Zhang ${ }^{1,13}$, Yong Zhang ${ }^{1,3}$, B. Zhao $^{8}$, J. Zhao ${ }^{1,3}$, L. Zhao ${ }^{6,7}$, L.Z. Zhao ${ }^{14}$, S.P. Zhao ${ }^{13,22}$, F. Zheng ${ }^{33}$, Y. Zheng ${ }^{8}$, B. Zhou ${ }^{1,3}$, H. Zhou ${ }^{28}$, J.N. Zhou ${ }^{15}$, P. Zhou ${ }^{10}$, R. Zhou $^{9}$, X.X. Zhou ${ }^{8}$, C.G. Zhu ${ }^{22}$, F.R. Zhu ${ }^{8}$, H. Zhu ${ }^{17}$, K.J. Zhu ${ }^{1,2,3,6}$ and X. Zuo ${ }^{1,3}$

${ }^{1}$ Key Laboratory of Particle Astrophyics \& Experimental Physics Division \& Computing Center, Institute of High Energy Physics, Chinese Academy of Sciences, 100049 Beijing, China.

${ }^{2}$ University of Chinese Academy of Sciences, 100049 Beijing, China.

${ }^{3}$ TIANFU Cosmic Ray Research Center, Chengdu, Sichuan, China.

${ }^{4}$ Dublin Institute for Advanced Studies, 31 Fitzwilliam Place, 2 Dublin, Ireland.

${ }^{5}$ Max-Planck-Institut for Nuclear Physics, P.O. Box 103980, 69029 Heidelberg, Germany.

${ }^{6}$ State Key Laboratory of Particle Detection and Electronics, China.

${ }^{7}$ University of Science and Technology of China, 230026 Hefei, Anhui, China.

${ }^{8}$ School of Physical Science and Technology \& School of Information Science and Technology, Southwest Jiaotong University, 610031 Chengdu, Sichuan, China.

${ }^{9}$ College of Physics, Sichuan University, 610065 Chengdu, Sichuan, China.

${ }^{10}$ School of Astronomy and Space Science, Nanjing University, 210023 Nanjing, Jiangsu, China.

${ }^{11}$ Center for Astrophysics, Guangzhou University, 510006 Guangzhou, Guangdong, China.

${ }^{12}$ School of Physics and Technology, Wuhan University, 430072 Wuhan, Hubei, China.

${ }^{13}$ Key Laboratory of Dark Matter and Space Astronomy, Purple Mountain Observatory, Chinese Academy of Sciences, 210023 Nanjing, Jiangsu, China.

${ }^{14}$ Hebei Normal University, 050024 Shijiazhuang, Hebei, China.

${ }^{15}$ Key Laboratory for Research in Galaxies and Cosmology, Shanghai Astronomical Observatory, Chinese Academy of Sciences, 200030 Shanghai, China.

${ }^{16}$ Key Laboratory of Cosmic Rays (Tibet University), Ministry of Education, 850000 Lhasa, Tibet, China.

${ }^{17}$ National Astronomical Observatories, Chinese Academy of Sciences, 100101 Beijing, China.

${ }^{18}$ School of Physics and Astronomy \& School of Physics (Guangzhou), Sun Yat-sen University, 519000 Zhuhai, Guangdong, China.

${ }^{19}$ Dipartimento di Fisica dell'Università di Napoli 'Federico II', Complesso Universitario di Monte Sant’Angelo, via Cinthia, 80126 Napoli, Italy

${ }^{20}$ School of Physics and Astronomy, Yunnan University, 650091 Kunming, Yunnan, China.

${ }^{21}$ Departement de Physique Nucleaire et Corpusculaire, Faculte de Sciences, Universite de Genève, 24 Quai Ernest Ansermet, 1211 Geneva, Switzerland.

${ }^{22}$ Institute of Frontier and Interdisciplinary Science, Shandong University, 266237 Qingdao, Shandong, China.

${ }^{23}$ Department of Engineering Physics, Tsinghua University, 100084 Beijing, China.

${ }^{24}$ School of Physics and Microelectronics, Zhengzhou University, 450001 Zhengzhou, Henan, China.

${ }^{25}$ Institute for Nuclear Research of Russian Academy of Sciences, 117312 Moscow, Russia. 
${ }^{26}$ School of Physics, Peking University, 100871 Beijing, China.

${ }^{27}$ School of Physical Science and Technology, Guangxi University, 530004 Nanning, Guangxi, China.

${ }^{28}$ Tsung-Dao Lee Institute \& School of Physics and Astronomy, Shanghai Jiao Tong University, 200240 Shanghai, China.

${ }^{29}$ Yunnan Observatories, Chinese Academy of Sciences, 650216 Kunming, Yunnan, China.

${ }^{30}$ Department of Physics, Faculty of Science, Mahidol University, 10400 Bangkok, Thailand.

${ }^{31}$ Moscow Institute of Physics and Technology, 141700 Moscow, Russia.

${ }^{32}$ Key Laboratory of Radio Astronomy, Purple Mountain Observatory, Chinese Academy of Sciences, 210023 Nanjing, Jiangsu, China.

${ }^{33}$ National Space Science Center, Chinese Academy of Sciences, 100190 Beijing, China. 